hep-ph/9807400

LA-UR-98-3047

\title{
Scalar Glueball Mixing and Decay
}

\author{
Leonid Burakovsky* and Philip R. Page ${ }^{\dagger}$ \\ Theoretical Division, MS B283 \\ Los Alamos National Laboratory \\ Los Alamos, NM 87545, USA
}

\begin{abstract}
We provide the first explanation of the counter-intuitive scalar glueball couplings to pseudoscalar mesons found in lattice QCD and predict hitherto uncalculated decay modes. Significant $a_{1} \pi$ and $(\pi \pi)_{S}(\pi \pi)_{S}$ couplings are found. We demonstrate the equivalence of linear and quadratic mass matrices for glueballquarkonium mixing. The equivalence of formalisms which deal with a glueballquarkonium basis and only a quarkonium basis is demonstrated. We show that the $f_{0}(1500)$ is not the heaviest state arising from glueball-quarkonium mixing for a glueball mass consistent with lattice QCD. The masses and couplings of scalar mesons, as well as their valence content, are calculated.
\end{abstract}

Key words: scalar glueball, scalar mesons, glueball decay, glueball dominance PACS: 12.39.Mk, 12.40.Yx, 13.25.Jx, 14.40.-n

${ }^{*}$ E-mail: BURAKOV@T5.LANL.GOV

${ }^{\dagger}$ E-mail: PRP@T5.LANL.GOV 


\section{Introduction}

The existence of a gluon self-coupling in QCD suggests that, in addition to the conventional $q \bar{q}$ states, there may be non $-q \bar{q}$ mesons: bound states built from gluons, called glueballs. The abundance of $q \bar{q}$ meson states in the $1-2 \mathrm{GeV}$ region and the possibility of glueball-quarkonium mixing makes the identification of the would-be lightest non $-q \bar{q}$ mesons extremely difficult. To date, no glueball state has been firmly established, although the existence of glueballs has been established in lattice QCD.

Although the current situation with the identification of glueball states is rather complicated, some progress has been made recently in the scalar glueball sector, where both experimental and lattice QCD results seem to converge. Various lattice QCD glueball mass estimates have been made in the literature, and one of the differences stems from the way the physical results are obtained from the raw lattice data: either by calculating the sting tension, or the $\rho$ mass. UKQCD estimates $1.55 \pm 0.05 \mathrm{GeV}$ by fixing to the string tension [1]. GF11 originally estimated $1.74 \pm 0.07 \mathrm{GeV}$ [2] by fixing to the $\rho$ mass. Later estimates include $1.71 \pm 0.06 \mathrm{GeV}$ [3] and $1.65 \pm 0.06 \mathrm{GeV}$ [1]. Attempts at reconciling UKQCD and GF11 results yielded $1.57 \pm 0.09$ [5] and $1.63 \pm 0.09$ [3]. In what follows, we shall take the glueball mass $m_{G}$ to be $1.6 \mathrm{GeV}$. Accordingly, there are two experimental candidates [6], $f_{0}(1500)$ and $f_{J}(1710)$, in the right mass range.

Recently, ref. [7] showed that the hypothesis where isoscalar meson mixing proceeds through an intermediate glueball, called "glueball dominance", can consistently account for isoscalar meson masses in various $J^{P C}$ sectors by employing glueball masses predicted by lattice QCD. Here we explore in detail the consequences of glueball dominance in the scalar sector, which differs from any other $J^{P C}$ sector due to the relative proximity of the glueball and quarkonia masses. Particularly, we demonstrate that the formulation of glueball dominance in refs. [7, 8] is consistent with glueball-quarkonium mixing formulated in refs. [3, 4, 9, 10]. Moreover, the recent lattice QCD calculation of ref. [1] found an unusual decay pattern for the scalar glueball, which is not consistent with any published model. We demonstrate that glueball dominance can explain this decay pattern.

In Section 2 the canonical formulation of glueball dominance is introduced. Section 
3 merges glueball dominance and the ${ }^{3} P_{0} /$ flux-tube model to describe glueball decays. Section 4 discusses glueball-quarkonium mixing. Phenomenological implications are indicated in Section 5, and a summary given in Section 6 .

\section{Glueball dominance}

We assume the glueball dominance of quarkonium mixing, viz., there is no direct quarkonium-quarkonium mixing, and the $q \bar{q} \leftrightarrow q^{\prime} \bar{q}^{\prime}$ transition is dominated by the glueball with the corresponding quantum numbers in the intermediate state [12].

Although the validity of glueball dominance has not been shown in QCD, the conclusions drawn from glueball dominance often agree with conclusions derived from the large number of colors $N_{c}$ limit of QCD:

- Consider a Feynman graph where a $q \bar{q}$ pair annihilates into an arbitrary number of gluons which then create a $q \bar{q}$ pair. In the large $N_{c}$ limit the graph is $\mathcal{O}\left(\frac{1}{N_{c}}\right)$ independent of the number of intermediate gluons. This corresponds to the finding (in glueball dominance) that the coupling between mesons via an intermediate glueball is largely independent of the $\mathrm{C}$-parity of the glueball [7], i.e. the number of gluons that a glueball can be built from in perturbative QCD.

- Consider a Feynman graph where (a) two gluons each create a $q \bar{q}$ pair (i.e. two quark loops) which again combines into two gluons; (b) two gluons combine to a single intermediate quark loop and then combine to two gluons. (a) is $\mathcal{O}\left(\frac{1}{N_{c}^{2}}\right)$ and (b) is $\mathcal{O}\left(\frac{1}{N_{c}}\right)$. Glueball dominance postulates that glueballs mix via a single intermediate meson, corresponding to (b), in agreement with the large $N_{c}$ limit.

- The glueball dominance description of glueball decay postulates that the glueball couples to a meson, which then subsequently decays. In order for this process to happen, the time $\tau$ for the $q \bar{q}$ pair created in the glueball to form a meson should be significantly less than the time $1 / \Gamma$ required for yet another $q \bar{q}$ pair to form so that the meson decays. In the large $N_{c}$ limit, $1 / \Gamma$ is $\mathcal{O}\left(N_{c}\right)$. The time taken for the created $q \bar{q}$ pair in the glueball to form a meson should be inversely proportional to the mass of the state, so that $\tau$ is $\mathcal{O}(1)$. Hence the 
large $N_{c}$ limit gives the condition $\tau \ll 1 / \Gamma$ required by the glueball dominance. Another way to see the large $N_{c}$ result is to note that quark pair creation is suppressed $\left(\mathcal{O}\left(\frac{1}{N_{c}}\right)\right)$ but quark rearrangement is not $(\mathcal{O}(1))$, so that created quarks in a glueball would rearrange to form a meson, rather than create another $q \bar{q}$ pair.

Here we review the glueball dominance picture discussed in more detail in ref. [7]. The possibility of the transition $q \bar{q} \rightarrow g g \ldots g \rightarrow q^{\prime} \bar{q}^{\prime}$ is accounted for by the quark mixing amplitudes, $A_{q q^{\prime}}$, which are included in the meson mass square matrix (written down here in the $s \bar{s},(u \bar{u}+d \bar{d}) / \sqrt{2}$ basis):

$$
\left(\begin{array}{cc}
m_{s \bar{s}}^{2}+A_{s s} & \sqrt{2} A_{s n} \\
\sqrt{2} A_{n s} & m_{n \bar{n}}^{2}+2 A_{n n}
\end{array}\right)
$$

where $m_{s \bar{s}}$ and $m_{n \bar{n}}, n=u, d$ are the primitive (bare) quarkonia masses.

The quark mixing amplitudes can be represented in the standard form,

$$
A \sim(\text { Vertex } 1) \cdot(\text { Propagator }) \cdot(\text { Vertex } 2)
$$

which reduces to

$$
A_{q q^{\prime}}=\sum_{i} \frac{\left\langle q \bar{q}\left|H_{p . c .}^{q^{\prime} \bar{q}^{\prime}}\right| i\right\rangle\left\langle i\left|H_{p . c .}^{q \bar{q}}\right| q^{\prime} \bar{q}^{\prime}\right\rangle}{M^{2}\left(q^{\prime} \bar{q}^{\prime}\right)-M^{2}(i)}
$$

where $H_{p . c .}^{q \bar{q}}$ is the quark pair creation operator for the flavor $q$, and $|i\rangle$ is a complete set of the (gluon) intermediate states. Because of the assumed glueball dominance, the sum (2.2) is saturated by the low-lying glueball:

$$
A_{q q} \simeq \frac{f_{q \bar{q} G}^{2}}{m_{q \bar{q}}^{2}-m_{\tilde{G}}^{2}},\left.\quad f_{q \bar{q} G} \equiv\left\langle q \bar{q}\left|H_{p . c .}^{q \bar{q}}\right| G\right\rangle\right|_{p^{\mu} p_{\mu}=m_{q \bar{q}}^{2}}
$$

for $q=n(=u, d), s$, and, in view of the factorization hypothesis discussed in more detail in ref. [7],

$$
A_{s n}=A_{n s} \equiv \sqrt{A_{n n} \cdot A_{s s}} \simeq \frac{f_{n \bar{n} G} f_{s \bar{s} G}}{\sqrt{\left(m_{n \bar{n}}^{2}-m_{\tilde{G}}^{2}\right)\left(m_{s \bar{s}}^{2}-m_{\tilde{G}}^{2}\right)}},
$$

where $f_{q \bar{q} G}$ defined in (2.3) is the coupling of the intermediate glueball to $q \bar{q}$, and $m_{\tilde{G}}$ is the corresponding (physical) glueball mass. 
Thus, the mass matrix $(2.1)$ reduces to

$$
\left(\begin{array}{cc}
m_{s \bar{s}}^{2}+\frac{f_{s \bar{s} G}^{2}}{m_{s \bar{s}}^{2}-m_{\tilde{G}}^{2}} & \frac{\sqrt{2} f_{n \bar{n} G} f_{s \bar{s} G}}{\sqrt{\left(m_{n \bar{n}}^{2}-m_{\tilde{G}}^{2}\right)\left(m_{s \bar{s}}^{2}-m_{\tilde{G}}^{2}\right)}} \\
\frac{\sqrt{2} f_{n \bar{n} G} f_{s \bar{s} G}^{2}}{\sqrt{\left(m_{n \bar{n}}^{2}-m_{\tilde{G}}^{2}\right)\left(m_{s \bar{s}}^{2}-m_{\tilde{G}}^{2}\right)}} & m_{n \bar{n}}^{2}+\frac{2 f_{n \bar{n} G}^{2}}{m_{n \bar{n}}^{2}-m_{\tilde{G}}^{2}}
\end{array}\right) .
$$

The masses of the physical isoscalar states $f_{0}$ and $f_{0}^{\prime}$ are obtained by diagonalizing this mass matrix:

$$
\left(\begin{array}{cc}
m_{f_{0}^{\prime}}^{2} & 0 \\
0 & m_{f_{0}}^{2}
\end{array}\right) .
$$

\section{Glueball decay}

\subsection{Scalar glueball decay to two pseudoscalar mesons}

Glueball decay via glueball dominance was introduced in ref. [13. We follow their approach, except for one improvement. Ref. [13] coupled the decaying glueball to an off-shell meson, which then subsequently decays to the outgoing mesons. The coupling used for this latter process is extracted from experiment where the off-shell meson is on-shell. This should not introduce undue errors unless the coupling is strongly dependent on energy of the off-shell meson.

In this section we shall deal with a case where the coupling is strongly dependent on the energy of the off-shell meson. Our proposed solution is to calculate the decay for the correct energy of the off-shell meson by employing the phenomenologically successful ${ }^{3} P_{0}$ model [14, 15]. Since the nonrelativistic ${ }^{3} P_{0}$ and Isgur-Paton fluxtube decay model give identical predictions for simple harmonic oscillator meson wave functions [16], which we employ, our predictions can also be viewed as predictions of the flux-tube model.

In fact, the flux-tube model, motivated from the strong coupling limit of the Hamiltonian formulation of the lattice gauge theory (HLGT), added to glueball dominance affords an intuitive picture of the decay process. In the lowest order in perturbation theory glueballs can be viewed as rings of flux in HLGT. Pair creation occurs in the first order of perturbation theory and breaks the flux-ring up into a meson. The flux-tube connecting the two quarks in the meson then breaks via the creation of a quark-antiquark pair with vacuum $\left({ }^{3} P_{0}\right)$ quantum numbers to form two outgoing 
mesons [17].

The amplitude (in $\mathrm{GeV}$ ) for the decay of the scalar glueball to two outgoing mesons is then given by

$$
\mathcal{M}=\frac{\sqrt{2} f_{n \bar{n} G}}{m_{n \bar{n}}^{2}-m_{\tilde{G}}^{2}} \mathcal{A}(n \bar{n})+\frac{f_{s \bar{s} G}}{m_{s \bar{s}}^{2}-m_{\tilde{G}}^{2}} \mathcal{A}(s \bar{s}),
$$

where $f_{n \bar{n} G}$ and $f_{s \bar{s} G}$ are the couplings of the scalar glueball to the intermediate $n \bar{n}$ and $s \bar{s}$ scalar mesons, respectively, introduced in Section 2. We have also taken care to insert the scalar meson propagator with the masses ordered according to the prescription of glueball dominance (2.5). $\mathcal{A}(n \bar{n})$ and $\mathcal{A}(s \bar{s})$ are the intermediate scalar meson ${ }^{3} P_{0}$ model decay amplitudes to two outgoing mesons. We have assumed that only the ground state scalar mesons saturate the decay.

The full ${ }^{3} P_{0}$ model amplitude is given in Appendix B of ref. [14; here we just write down the case of identical inverse radii $\beta_{A}=\beta_{B}=\beta_{C} \equiv \beta$ for identical quark masses ${ }^{1}$, for simplicity (A denotes the scalar meson, and B and $\mathrm{C}$ the outgoing mesons):

$$
\mathcal{A}=\sqrt{\frac{8 m_{G}^{2} \tilde{M}_{B} \tilde{M}_{C}}{\tilde{M}_{A}}} \frac{16 \pi^{\frac{3}{4}}}{9 \sqrt{\beta}} \gamma_{0}\left(1-\frac{2 p^{2}}{9 \beta^{2}}\right) \exp \left\{-\frac{p^{2}}{12 \beta^{2}}\right\},
$$

where we neglected the factor arising from the flavors of the mesons. The mock meson phase space convention is specified by $\tilde{M}_{A, B, C}$ [14, and $p$ is the momentum of the outgoing meson $\mathrm{B}$ in the glueball rest frame. The width is computed from the amplitude in Eq. (3.2) by using the standard formula given by the Particle Data Group [6]. The pair creation constant $\gamma_{0}$ is usually taken to be the same for $n \bar{n}$ and $s \bar{s}$ pair creation [14, 18], as we shall do here.

The composition of $\eta$ and $\eta^{\prime}$ : is

$$
\eta=\sin \theta|n \bar{n}\rangle+\cos \theta|s \bar{s}\rangle, \quad \eta^{\prime}=\cos \theta|n \bar{n}\rangle-\sin \theta|s \bar{s}\rangle
$$

We obtain the following simple relationships between the ${ }^{3} P_{0}$ model amplitudes when $\beta_{A}, \beta_{B}, \beta_{C}, p$ and the mock meson phase space are taken to be constant for all meson decay processes:

$$
\mathcal{A}\left(\frac{u \bar{u}+d \bar{d}}{\sqrt{2}} \rightarrow \pi \pi\right): \mathcal{A}\left(\frac{u \bar{u}+d \bar{d}}{\sqrt{2}} \rightarrow K \bar{K}\right): \mathcal{A}\left(\frac{u \bar{u}+d \bar{d}}{\sqrt{2}} \rightarrow \eta \eta\right):
$$

\footnotetext{
${ }^{1}$ The ${ }^{3} P_{0}$ model amplitudes depend explicitly on the light and strange quark masses for decays where the initial quarks are different from the quarks in the created pair. In this work we take the light and strange quark masses to be identical for decays to $K \bar{K}$.
} 


$$
\begin{aligned}
& \mathcal{A}\left(\frac{u \bar{u}+d \bar{d}}{\sqrt{2}} \rightarrow \eta \eta^{\prime}\right): \mathcal{A}(s \bar{s} \rightarrow K \bar{K}): \mathcal{A}(s \bar{s} \rightarrow \eta \eta): \mathcal{A}\left(s \bar{s} \rightarrow \eta \eta^{\prime}\right) \\
& =2: 1: 2 \sin ^{2} \theta: 2 \sin \theta \cos \theta: \sqrt{2}: 2 \sqrt{2} \cos ^{2} \theta:-2 \sqrt{2} \sin \theta \cos \theta
\end{aligned}
$$

When we take $\frac{f_{n \bar{n} G}}{m_{\tilde{G}}^{2}-m_{n \bar{n}}^{2}}=\frac{f_{s \bar{s} G}}{m_{\tilde{G}}^{2}-m_{s \bar{s}}^{2}}$ (explained below), we obtain

$$
\begin{gathered}
\mathcal{M}(G \rightarrow \pi \pi): \mathcal{M}(G \rightarrow K \bar{K}): \mathcal{M}(G \rightarrow \eta \eta): \mathcal{M}\left(G \rightarrow \eta \eta^{\prime}\right)= \\
1: 1: 1: 0 .
\end{gathered}
$$

This is the result one obtains when naïvely coupling the quarks in the outgoing mesons to the vacuum [19], often referred to as "flavour democratic coupling". It would also yield a horizontal line for our predicted amplitude in Fig. 1.

The lattice results were obtained in the $S U(3)$ limit. To compare we shall also adopt the $S U(3)$ limit in the remainder of this section. Hence we take the couplings and quark masses to be identical, i.e. $f_{n \bar{n} G}=f_{s \bar{s} G} \equiv f_{S U(3) G}$ and $m_{n \bar{n}}=m_{s \bar{s}} \equiv m_{S U(3)}$.

Fig. 1 shows our results.

The solid line represents our basic prediction. We use $\beta=0.4 \mathrm{GeV}$ found to enable a fit of a large range of meson decays [14, 18]. Mock meson phase space is employed since this enables a prediction of $\mathcal{M}$ for all $p$, as can be done in lattice QCD. Since we work in the limit of $S U(3)$ symmetry, we take the mock meson phase space parameters to be those of say $s \bar{s}$ mesons, i.e. $\tilde{M}_{A}=1.49 \mathrm{GeV}, \tilde{M}_{B}, \tilde{M}_{C}=0.85$ $\mathrm{GeV}$ (see Table 5 of ref. [14). We take the pair creation constant to be $\gamma_{0}=0.39$ [14.

Since all parameters are constrained, except for $f_{S U(3) G} /\left(m_{S U(3)}^{2}-m_{\tilde{G}}^{2}\right)$, we regard our fit in Fig. 1 as a one-parameter fit. This parameter sets the overall decay strength of the glueball, and does not influence the relative strengths of the various decay modes.

The lattice results $]$ for the $\eta \eta, K \bar{K}$ and $\pi \pi$ decay modes are plotted from left to right as the data points. It is non-trivial that our results are consistent. The fact that the prediction in Fig. 1 is not a horizontal line, as one naïvely expects, indicates that

\footnotetext{
${ }^{2}$ GF11 predicts $\frac{\mathcal{M}}{m_{\rho}}=0.834_{-0.579}^{+0.603}, 2.654_{-0.402}^{+0.372}, 3.099_{-0.423}^{+0.364}$ for scalar glueball decay to $\pi \pi, K \bar{K}$ and $\eta \eta$, respectively [11, 20], where $m_{\rho}$ is the mass of the $\rho$ meson. The predictions are given as a function of pseudoscalar mass, which we translate to the momentum of the outgoing mesons $p$ using conservation of energy: $p^{2}=m_{G}^{2} / 4-m_{P S}^{2}$, where $m_{P S}$ is the relevant pseudoscalar meson mass.
} 
the detailed dynamics of the flux-tube and ${ }^{3} P_{0}$ models combined with the hypothesis of glueball dominance captures the correct strong interaction dynamics. This success is not shared by other models of glueball decay based on perturbative QCD decay dynamics, where the naïve pattern of Eq. (3.5) arises [21]. The other points and lines in Fig. 1 indicate parameter variations and are discussed in the caption of the figure.

We fit

$$
\left|\frac{f_{S U(3) G}}{m_{\tilde{G}}^{2}-m_{S U(3)}^{2}}\right|=0.34 \pm 0.04 .
$$

\subsection{Scalar glueball decay to two mesons}

Having predicted the scalar glueball decay to pseudoscalar mesons, we are now in a position to make the first predictions in the literature of the decay of the scalar glueball to non-pseudoscalar mesons.

Since we have fitted Eq. (3.6) using mock meson phase space, we again use this convention and hence $\gamma_{0}=0.39$ [14]. We again use $\beta=0.4 \mathrm{GeV}$ and do the calculation in the $S U(3)$ limit with the $s \bar{s}$ mock meson masses [1] [14]. The results are indicated in Table 1. The primitive glueball amplitudes should be understood to be correct up to a sign. The analytical expressions used for the amplitudes can be found in Appendix A.

We see from Table 1 that the total width of the $1.6 \mathrm{GeV}$ scalar glueball is $250-390$ MeV excluding $(\pi \pi)_{S}(\pi \pi)_{S}$ decays. There is also substantial phase space dependence for the glueball decay amplitudes to $\pi \pi, K \bar{K}, \eta \eta$ and $\pi(1300) \pi$.

\section{Glueball mixing}

\subsection{Glueball-quarkonium basis}

\footnotetext{
${ }^{3}$ Taking the parameters for $n \bar{n}$ mesons, i.e. $\tilde{M}_{A}=1.25 \mathrm{GeV}, \tilde{M}_{B}, \tilde{M}_{C}=0.85 \mathrm{GeV}$ 14 would give scalar meson widths to $\pi \pi, K \bar{K}$ and $\eta \eta \sim 20 \%$ larger. A fit to the lattice data then yields a different value for $f_{S U(3) G} /\left(m_{S U(3)}^{2}-m_{\tilde{G}}^{2}\right)$. The predictions for the glueball widths to $\pi \pi, K \bar{K}$ and $\eta \eta$ are identical, and the dominant width in Table 1, to $a_{1} \pi$, is $1 \%$ different from the value quoted.
} 


\subsubsection{Glueball-quarkonium linear mass matrix}

In ref. [10], Weingarten suggested the following $3 \times 3$ linear mass matrix, which stems from the Hamiltonian formulation of QCD, to describe the mixing of a glueball and quarkonia:

$$
\left(\begin{array}{ccc}
m_{G} & z & \sqrt{2} z \\
z & m_{s \bar{s}} & 0 \\
\sqrt{2} z & 0 & m_{n \bar{n}}
\end{array}\right),
$$

where $z$ stands for the annihilation amplitude of quarkonium into a glueball which has dimensionality (mass) and represents a counterpart of our $f$ 's which have dimensionality (mass) ${ }^{2}$. In order to test our results by comparing with available lattice QCD data, we should establish a relation between this linear mass matrix and our mass squared one.

\subsubsection{Glueball-quarkonium quadratic mass matrix}

We first rewrite Weingarten's matrix for the squares of the glueball and quarkonia masses and show its equivalence to glueball dominance in the $2 \times 2$ subspace spanned by quarkonia. We then establish a relation between Weingarten's linear and our quadratic mass matrices.

So, consider

$$
\left(\begin{array}{ccc}
m_{G}^{2} & f & \sqrt{2} f \\
f & m_{s \bar{s}}^{2} & 0 \\
\sqrt{2} f & 0 & m_{n \bar{n}}^{2}
\end{array}\right)
$$

where the vanishing off--diagonal elements indicate that there is no direct quarkoniumquarkonium mixing, i.e., glueball dominance.

Proposition 1. The mass matrix (4.2) is equivalent (gives the same physical quarkonia masses) to glueball dominance in the $2 \times 2$ subspace spanned by quarkonia (with $f_{n \bar{n} G}=f_{s \bar{s} G}$ ).

Proof. First, we rewrite the mass matrix (2.5) (with $f_{n \bar{n} G}=f_{s \bar{s} G}$ ) in the following form:

$$
\left(\begin{array}{cc}
m_{s \bar{s}}^{2}-r^{2} A & \sqrt{2} r A \\
\sqrt{2} r A & m_{n \bar{n}}^{2}-2 A
\end{array}\right), \quad A \equiv \frac{f_{n \bar{n} G}^{2}}{m_{\tilde{G}}^{2}-m_{n \bar{n}}^{2}}, \quad r \equiv \sqrt{\frac{m_{\tilde{G}}^{2}-m_{n \bar{n}}^{2}}{m_{\tilde{G}}^{2}-m_{s \bar{s}}^{2}}}
$$


where $r$ is a complex number. The masses of the two physical states are now determined from the equations

$$
\begin{aligned}
m_{f_{0}}^{2}+m_{f_{0}^{\prime}}^{2} & =m_{n \bar{n}}^{2}+m_{s \bar{s}}^{2}-A\left(2+r^{2}\right) \\
m_{f_{0}}^{2} m_{f_{0}^{\prime}}^{2} & =m_{n \bar{n}}^{2} m_{s \bar{s}}^{2}-A\left(2 m_{s \bar{s}}^{2}+r^{2} m_{n \bar{n}}^{2}\right) .
\end{aligned}
$$

We take the equivalence of the matrices (2.5) and (4.2) to mean the equality of the corresponding eigenvalues $m_{f_{0}}^{2}, m_{f_{0}^{\prime}}^{2}$.

The eigenvalues of (4.2) are determined from the following three equations:

$$
\begin{aligned}
m_{\tilde{G}}^{2}+m_{f_{0}}^{2}+m_{f_{0}^{\prime}}^{2} & =m_{G}^{2}+m_{n \bar{n}}^{2}+m_{s \bar{s}}^{2} \\
m_{\tilde{G}}^{2} m_{f_{0}}^{2}+m_{\tilde{G}}^{2} m_{f_{0}^{\prime}}^{2}+m_{f_{0}}^{2} m_{f_{0}^{\prime}}^{2} & =m_{G}^{2} m_{n \bar{n}}^{2}+m_{G}^{2} m_{s \bar{s}}^{2}+m_{n \bar{n}}^{2} m_{s \bar{s}}^{2}-3 f^{2}, \\
m_{\tilde{G}}^{2} m_{f_{0}}^{2} m_{f_{0}^{\prime}}^{2} & =m_{G}^{2} m_{n \bar{n}}^{2} m_{s \bar{s}}^{2}-f^{2}\left(2 m_{s \bar{s}}^{2}+m_{n \bar{n}}^{2}\right)
\end{aligned}
$$

It then follows from Eqs. (4.4),(4.6) and Eqs. (4.5),(4.8) that:

$$
f^{2}=\left(\frac{2 m_{s \bar{s}}^{2}+r^{2} m_{n \bar{n}}^{2}}{2 m_{s \bar{s}}^{2}+m_{n \bar{n}}^{2}} m_{\tilde{G}}^{2}-\frac{\left(2+r^{2}\right) m_{n \bar{n}}^{2} m_{s \bar{s}}^{2}}{2 m_{s \bar{s}}^{2}+m_{n \bar{n}}^{2}}\right) A
$$

and from Eqs. (4.4)-(4.7):

$$
f^{2}=\left(\frac{2+r^{2}}{3} m_{\tilde{G}}^{2}-\frac{2 m_{n \bar{n}}^{2}+r^{2} m_{s \bar{s}}^{2}}{3}\right) A .
$$

Using the definition of $r$ in Eq. (4.3), the equivalence of Eqs. (4.9) and (4.10) follows by simple algebra. Also, using the definition of $A$ in Eq. (4.3), and inserting it in either Eqs. (4.9) or (4.10), we obtain that $\pm f=f_{n \bar{n} G}=f_{s \bar{s} G}$.

This important result means that glueball dominance is nothing else but an effective representation of the glueball-quarkonia mixing in the $2 \times 2$ subspace spanned by quarkonia. The relation is only possible because both formulations descibe the physics in terms of the same basis states.

It is natural to define $f \equiv f_{n \bar{n} G}=f_{s \bar{s} G}$, noting that Eq. (4.2) assumes $S U(3)$ symmetric couplings. This definition is consistent with what is obtained when the equivalence of the $2 \times 2$ and $3 \times 3$ formalisms is demanded in Proposition 1 .

The mass matrix (4.2) possesses, however, more generality than the naïve glueball dominance picture in the $2 \times 2$ quarkonia subspace. This is because the former, in contrast to the latter, allows one to obtain the valence glue content of the physical quarkonia, and the valence content of the physical glueball. 
Proposition 2. The linear, (4.1), and quadratic, (4.2), formulations for the scalar mesons are equivalent provided that (i) $z^{2} \ll m_{G}^{2}, m_{n \bar{n}}^{2}, m_{s \bar{s}}^{2}$, (ii) $m_{s \bar{s}}-m_{n \bar{n}} \ll m_{n \bar{n}}, m_{s \bar{s}}$ or $m_{n \bar{n}}, m_{s \bar{s}} \ll m_{G}$.

Proof. We take the equivalence of the linear, (4.1), and quadratic, (4.2), formulations to mean (i) the equality of the eigenvalues of the matrix (4.2) to the eigenvalues squared of the matrix (4.1), (ii) the equality of the eigenvectors of both matrices, for the same values of the input parameters $m_{G}, m_{n \bar{n}}, m_{s \bar{s}}$. Denote the matrices (4.1),(4.2) by $M_{\text {lin }}$ and $M_{\text {qaud }}$, respectively, the corresponding diagonalized matrices by $\Lambda_{l i n}$ and $\Lambda_{\text {qaud }}$, and the matrix that diagonalizes the $M$ 's by $S$ (it is the same for $M_{l i n}$ and $M_{\text {qaud }}$ because both have by construction the same eigenvectors).

Since $\Lambda_{\text {lin }}=\operatorname{diag}\left(m_{\tilde{G}}, m_{f_{0}^{\prime}}, m_{f_{0}}\right)$,

$$
\Lambda_{\text {lin }}^{2}=\operatorname{diag}\left(m_{\tilde{G}}^{2}, m_{f_{0}^{\prime}}^{2}, m_{f_{0}}^{2}\right)=\Lambda_{q a u d}
$$

It follows from this relation and

$$
\Lambda_{\text {lin }}^{2}=S M_{l i n} S^{-1} \cdot S M_{l i n} S^{-1}=S M_{l i n}^{2} S^{-1}, \quad \Lambda_{q a u d}=S M_{q a u d} S^{-1}
$$

that

$$
M_{\text {qaud }}=M_{\text {lin }}^{2}
$$

Thus, the linear and quadratic formulations are equivalent provided that Eq. (4.11) is valid.

Since the square of the mass matrix (4.1) is

$$
\left(\begin{array}{ccc}
m_{G}^{2}+3 z^{2} & z\left(m_{G}+m_{s \bar{s}}\right) & \sqrt{2} z\left(m_{G}+m_{n \bar{n}}\right) \\
z\left(m_{G}+m_{s \bar{s}}\right) & m_{s \bar{s}}^{2}+z^{2} & \sqrt{2} z^{2} \\
\sqrt{2} z\left(m_{G}+m_{n \bar{n}}\right) & \sqrt{2} z^{2} & m_{n \bar{n}}^{2}+2 z^{2}
\end{array}\right),
$$

it is clear that Eq. (4.11) follows if both conditions (i) and (ii) of Proposition 2 are satisfied.

The equivalence of (4.2) and (4.12) also implies the following relation between $z$ and $f: f=z\left(m_{G}+m_{q \bar{q}}\right)$. In the scalar sector where the glueball and quarkonia have comparable masses, it reduces to

$$
f \simeq 2 z m_{G}
$$


For any other $J^{P C}$ multiplet, $m_{G} \gg m_{q \bar{q}}$, and, respectively, $f \simeq z m_{G}$.

An explicit numerical example on the equivalence of linear and quadratic mass matrix formulations is given in Appendix B.

\subsection{Scalar meson spectroscopy}

As the relation between Weingarten's linear mass matrix and our quadratic mass matrix is established in the previous subsection, we are ready to consider scalar meson spectroscopy implied by glueball dominance, and compare our results with the lattice QCD simulations of refs. [3], 10].

We shall first show that within the glueball dominance hypothesis, the $f_{0}(1500)$ cannot be the heaviest isoscalar scalar meson arising from ground state $n \bar{n}, s \bar{s}$ and glueball mixing if $m_{G}>1.5 \mathrm{GeV}$.

The argument is as follows. There are three possibilities: (i) $m_{s \bar{s}}<m_{G}$, (ii) $m_{s \bar{s}}>m_{G}$, (iii) $m_{s \bar{s}}=m_{G}$. The main property of the $3 \times 3$ mass matrices (4.1),(4.2) (we do not prove it here) is that upon mixing the higher mass primitive state becomes more massive, while the lower mass primitive state becomes less massive (i.e., the mass splitting between the higher and lower mass primitive states increases as a result of the mixing). Therefore, in the case (i) $m_{\tilde{G}}>m_{G}>1.5 \mathrm{GeV}$; in the case (ii) $m_{f_{0}^{\prime}}>m_{s \bar{s}}>m_{G}>1.5 \mathrm{GeV}$. Finally, in the case (iii) it can be shown that the

physical $s \bar{s}$ and glueball states have masses $\simeq \sqrt{m_{G}^{2} \pm f}$, and therefore, one of them is always higher than $m_{G}>1.5 \mathrm{GeV}$.

Hence, the $f_{0}(1500)$ is not the heaviest isoscalar scalar meson arising from ground state $n \bar{n}, s \bar{s}$ and glueball mixing. If both the existence of $f_{0}(980)$ and $f_{0}(1370) / f_{0}(400-$ 1200) are confirmed by experiment, there has to be an extra degree of freedom to account for the existence of these states.

\footnotetext{
${ }^{4}$ This conclusion may be modified by the inclusion of effects beyond glueball dominance, e.g., coupled channels 22], which may be especially relevant for scalar states [23. Ref. 22, finds that the masses of the states are always lower than those of the primitive states. We believe this to be an artifact of the inclusion of only low-lying channels, which could lead to misleading results 23].
} 


\subsubsection{The glueball-quarkonium coupling $f$}

In ref. [3], Lee and Weingarten estimate the mixing parameter $z$ to bef

$$
|z|=56 \pm 37 \mathrm{MeV} .
$$

Eq. (4.14) implies, via (4.13) with $m_{G}=1.65 \pm 0.06 \mathrm{GeV}$ 四,

$$
|f| \simeq 0.19 \pm 0.13 \mathrm{GeV}^{2} .
$$

This implies that in the flavor $S U(3)$ limit where we take $m_{S U(3)}=m_{n \bar{n}}=m_{s \bar{s}}=$ $m_{s \bar{n}}=m_{K_{0}^{*}}=1.43 \mathrm{GeV}$ [6] (and the same $m_{G}=1.65 \pm 0.06 \mathrm{GeV}$ ),

$$
\left|\frac{f}{m_{G}^{2}-m_{S U(3)}^{2}}\right|=0.28_{-0.21}^{+0.38},
$$

consistent with the value needed from glueball decay (Eq. (3.6)).

We take from Eq. (3.6), defining $m_{q \bar{q}}=\left(m_{n \bar{n}}+m_{s \bar{s}}\right) / 2$,

$$
\left|\frac{f}{m_{\tilde{G}}^{2}-m_{q \bar{q}}^{2}}\right|=0.34 \pm 0.04
$$

\subsubsection{Two simulations for glueball-quarkonium mixing}

We now wish to consider two simulations for the glueball-quarkonia mixing based on the quadratic mass matrix (4.2), and extract the masses of the primitive quarkonia and the physical states, with the help of Eqs. (4.15) and (4.17).

We use Eqs. (4.6)-(4.8) for the masses of the physical states $m_{\tilde{G}}, m_{f_{0}}, m_{f_{0}^{\prime}}$.

For the first simulation, we also employ Eq. (4.17) and

$$
m_{G}=1.6 \mathrm{GeV}, \quad m_{s \bar{s}}-m_{n \bar{n}}=250 \pm 50 \mathrm{MeV},
$$

the latter being a typical mass splitting] between $s \bar{s}$ and $n \bar{n}$ states for different meson

\footnotetext{
${ }^{5}$ In a most recent paper 汪, Lee and Weingarten introduce $S U(3)$ breaking effects, in terms of different values of $z$ for $G-n \bar{n}, G-s \bar{s}$ mixing, $z_{G n \bar{n}} / z_{G s \bar{s}}=1.198 \pm 0.072$, and estimate $\left|z_{G s \bar{s}}\right|=43 \pm 31$ $\mathrm{MeV}$, which implies, with the above ratio, $\left|z_{G n \bar{n}}\right|=54 \pm 40 \mathrm{MeV}$, similar to (4.14).

${ }^{6}$ Note that we could use the mass squared splitting for quarkonia, $m_{s \bar{s}}^{2}-m_{n \bar{n}}^{2}$, in place of the linear one in Eq. (4.18). With, e.g., the value for this mass squared splitting $0.65 \pm 0.01 \mathrm{GeV}^{2}$, since for the remaining three $P$-wave nonets $\left(\right.$ in $\left.\mathrm{GeV}^{2}\right) 2\left(m_{K_{2}^{*}}^{2}-m_{a_{2}}^{2}\right) \simeq 0.64,2\left(m_{K_{1 A}}^{2}-m_{a_{1}}^{2}\right) \simeq 0.66$, $2\left(m_{K_{1 B}}^{2}-m_{b_{1}}^{2}\right) \simeq 0.65$ [24], the solution is $m_{\tilde{G}}=1694 \pm 27 \mathrm{MeV}, m_{f_{0}}=1242 \pm 21 \mathrm{MeV}, m_{n \bar{n}}=$ $1314 \pm 12 \mathrm{MeV}, m_{s \bar{s}}=1542 \pm 8 \mathrm{MeV},|f|=0.285 \pm 0.06 \mathrm{GeV}^{2}$, and $\sqrt{\left(m_{n \bar{n}}^{2}+m_{s \bar{s}}^{2}\right) / 2}=1432 \pm 10$ $\mathrm{MeV}$, consistent with the case of the linear mass splitting (middle column, Table 2).
} 
multiplets [6, 24], and solve the system of 5 equations (4.6)-(4.8),(4.17),(4.18) for $m_{\tilde{G}}$, $m_{f_{0}}, m_{n \bar{n}}, m_{s \bar{s}}$ and $f$, by fixing $m_{f_{0}^{\prime}}=1.5 \mathrm{GeV}$.

The reason for the latter requirement is that $f_{0}(1500)$ is established in more decay channels that any other scalar meson, and we should therefore construct our simulation of scalar meson spectroscopy with the constraint that one of the masses of the physical states is $1503 \pm 11 \mathrm{MeV}$ [6].

For the second simulation, we employ Eq. (4.15) in place of (4.17). The solution to Eqs. (4.6)-(4.8),(4.17),(4.18) for the first case, and Eqs. (4.6)-(4.8),(4.15),(4.18) for the second case are presented in Table 2.

\begin{tabular}{|c||c||c|}
\hline & Eqs. (4.6)-(4.8),(4.17),(4.18) & Eqs. (4.6)-(4.8),(4.15),(4.18) \\
\hline$m_{\tilde{G}}, \mathrm{MeV}$ & $1703 \pm 40$ & $1649_{-41}^{+63}$ \\
\hline$m_{f_{0}}, \mathrm{MeV}$ & $1218 \pm 70$ & $1248_{-72}^{+52}$ \\
\hline$m_{s \bar{s}}, \mathrm{MeV}$ & $1546 \pm 17$ & $1527 \pm 23$ \\
\hline$m_{n \bar{n}}, \mathrm{MeV}$ & $1296 \pm 33$ & $1277 \pm 27$ \\
\hline$|f|, \mathrm{GeV}^{2}$ & $0.305 \pm 0.09$ & $0.19 \pm 0.13$ \\
\hline
\end{tabular}

Table 2: Solution to Eqs. (4.6)-(4.8),(4.17),(4.18), and Eqs. (4.6)-(4.8),(4.15),(4.18).

We note that the value of $f$ obtained in the first case is consistent with the value extracted from lattice QCD (Eq. (4.15)). It is also in agreement with values extracted phenomenologically for different $J^{P C}$ meson nonets in ref. [7] which all are in the interval $0.27-0.32 \mathrm{GeV}^{2}$. We however disagree with lattice QCD that the primitive $s \bar{s}$ is at least $200 \mathrm{MeV}$ below the primitive glueball [3, 4] but only $\simeq 70 \pm 30 \mathrm{MeV}$, as seen from our solutions for $m_{s \bar{s}}$ in the two cases considered. We also note that $\sqrt{\left(m_{n \bar{n}}^{2}+m_{s \bar{s}}^{2}\right) / 2}$ which is $1426 \pm 24$ and $1407 \pm 25 \mathrm{MeV}$ in the two cases, respectively, is consistent with $m_{K_{0}^{*}}=1429 \pm 6 \mathrm{MeV}$ [6].

\footnotetext{
${ }^{7}$ Note that, although we do find a solution with $m_{s \bar{s}}>m_{G}$ for both simulations, the values for $m_{n \bar{n}}$ and $m_{s \bar{s}}$ obtained are too high to be accommodated by any of the existing quark models; typically $m_{n \bar{n}} \sim 1.6 \mathrm{GeV}, m_{s \bar{s}} \sim 1.8 \mathrm{GeV}$. Also, $f_{0}(1500)$ is the lightest of the three scalars. Our results are therefore in agreement with the conclusion of ref. [3] that the situation where the primitive $s \bar{s}$ state has a higher mass than the primitive glueball is incompatible with lattice QCD.
} 
With $f>0$, the valence content of the three physical states obtained in the first simulation, is

$$
\begin{gathered}
|1703\rangle=(0.821 \pm 0.02)|G\rangle+(0.493 \pm 0.02)|s \bar{s}\rangle+(0.287 \pm 0.05)\left|\frac{u \bar{u}+d \bar{d}}{\sqrt{2}}\right\rangle \\
|1500\rangle=-(0.410 \pm 0.04)|G\rangle+(0.860 \pm 0.02)|s \bar{s}\rangle-(0.305 \pm 0.08)\left|\frac{u \bar{u}+d \bar{d}}{\sqrt{2}}\right\rangle \\
|1218\rangle=-(0.397 \pm 0.08)|G\rangle+(0.133 \pm 0.05)|s \bar{s}\rangle+(0.908 \pm 0.05)\left|\frac{u \bar{u}+d \bar{d}}{\sqrt{2}}\right\rangle
\end{gathered}
$$

and shows that the physical glueball contains $\sim 70 \%$ glue and $\sim 30 \% q \bar{q}$, while each of the physical quarkonia contains $\sim 15 \%$ glue and $\sim 85 \% q \bar{q}$. The overall signs for the states have no physical significance.

Although the masses of the physical states do not depend on the sign of $f$, the valence content of the physical states does. Namely, we find that under the inversion of the sign of $f$ both the quark content of the physical glueball and the glue content of the physical quarkonia change their sign. However, it is not difficult to see that the ${ }^{3} P_{0}$ model decay width of the physical states remains invariant under the inversion of the sign of $f$. Hence, for the study of masses and decays of scalar mesons, one need to consider the case $f>0$ only.

Notwithstanding the similarity of the results obtained, there is a principal difference between our approach and that of refs. [3, 4, 10]. Lee and Weingarten choose the input parameters $m_{G}, m_{s \bar{s}}, m_{n \bar{n}}$ and $z$ to obtain the three physical masses. Although the input parameters $m_{G}$ and $z$ obtained are consistent with their lattice QCD calculations, the mass splitting $m_{s \bar{s}}-m_{n \bar{n}} \sim 50 \mathrm{MeV}$, is atypical of that provided by constituent quark models in Eq. (4.18). Moreover, the Gell-Mann-Okubo type relation $m_{n \bar{n}}^{2}+m_{s \bar{s}}^{2}=2 m_{s \bar{n}}^{2}=2 m_{K_{0}^{*}}^{2}$ [24] does not hold in their approach. In contrast, our approach does not assume $m_{u \bar{u}}$ and $m_{s \bar{s}}$ individually, but only their difference. To compensate, we need one more input parameter: we require that one of the physical masses be in agreement with the well established scalar state $f_{0}(1500)$.

\section{Phenomenology}

For some time there has been a controversy over the spin $J$ assignment of $f_{J}(1710)$, and hence the existence of a scalar state at this mass [19]. This matter is not yet fully 
resolved. Our results in Section 4.2 suggest that $f_{0}(1500)$ cannot be the heaviest state arising from glueball-quarkonium mixing. Hence we highlight recent evidence for a $J=0$ component in $f_{J}(1710)$. BES seperated both a $J=0$ and a $J=2$ component, with the scalar state having mass $1780 \mathrm{MeV}$ and a width of $85 \pm 25 \mathrm{MeV}$ [25]. There are also claims of a $J=0$ signal at $1750 \pm 15 \mathrm{MeV}$ with width $160 \pm 40 \mathrm{MeV}$ [26], and with mass $1704_{-23}^{+16} \mathrm{MeV}$ with width $124_{-44}^{+52} \mathrm{MeV}$ [27]. $m_{\tilde{G}}$ obtained in Table 2 is consistent with these experimental masses.

The $a_{1} \pi$ decay of the primitive glueball is expected to be larger than any pseudoscalar decay mode. There is some evidence for the production of $f_{0}(1500)$ via $a_{1}^{+}$ exchange in the reaction $\pi^{-} p \rightarrow \pi^{+} \pi^{-} n$, i.e. an $a_{1} \pi$ coupling of the $f_{0}(1500)$, in CERN-Cracow-Munich data with a polarized target [28]. We urge experimenters to allow for the $a_{1} \pi$ decay mode in partial wave analyses. This applies to Crystal Barrel at CERN for analysis of $\pi^{+} \pi^{-} \pi^{0} \pi^{0}[29]$ and $\pi^{+} \pi^{-} \pi^{+} \pi^{-}$data, and to Mark III $\pi^{+} \pi^{-} \pi^{+} \pi^{-}$data [26].

The $(\pi \pi)_{S}(\pi \pi)_{S}$ decay of the glueball can be very substantial, depending sensitively on the width and mass of the intermediate $f_{0}$ coupling to $(\pi \pi)_{S}$ (see Table 1 ). There are indeed indications of substantial $(\pi \pi)_{S}(\pi \pi)_{S}$ decay modes in $f_{0}(1500)$ and $f_{0}(1710)$ [6, 26, 30].

The two-photon widths of the various states provide stringent consistency checks for our results. In the flavour $S U(3)$ limit the $\gamma \gamma$ width for a state $\alpha_{G}|G\rangle+\alpha_{s \bar{s}}|s \bar{s}\rangle+$ $\alpha_{n \bar{n}}\left|\frac{u \bar{u}+d \bar{d}}{\sqrt{2}}\right\rangle$ is proportional to

$$
\left(\alpha_{s \bar{s}}+\frac{5}{\sqrt{2}} \alpha_{n \bar{n}}\right)^{2}
$$

where we have taken the charges of the quarks into account, and normalized the expression to be unity when the state is built purely from $s \bar{s}$.

For the states in Eqs. (4.19)-(4.21) we obtain the two-photon width ratio for the states $f_{0}(1370), f_{0}(1500), f_{0}(1710)$ to be $11: 0.05: 2$.

If one takes model-dependent estimates of two-photon widths of $s \bar{s}$, i.e. $0.16-$ $0.20 \mathrm{keV}$ [31], one observes that the two-photon width of $f_{0}(1500)$ is consistent with the experimental bound of $<0.17 \mathrm{keV}$ [32]. Since $f_{0}(1370)$ is dominated by light quarks, our estimate for the two-photon width of $f_{0}(1370)$ is $\frac{2}{25} 11$ times the twophoton width of $\frac{u \bar{u}+d \bar{d}}{\sqrt{2}}(3.25-6.46 \mathrm{keV}$ [31]). This is consistent $5.4 \pm 2.3 \mathrm{keV}$ [6] from 
experiment. There are currently no experimental estimates for $\gamma \gamma$ width of the $J=0$ component of $f_{J}(1710)$.

From Eqs. (4.19)-(4.21) it is clear that the glueball content of $f_{J}(1710)(J=0)$ is significantly higher than that of $f_{0}(1500)$ and $f_{0}(1370)$, which are similar. This can be tested by evaluating the states' coupling to two gluons in the model of ref. [19]: $B R\left(f_{0}(1710) \rightarrow g g\right) \geq 0.5$ [33] and $B R\left(f_{0}(1500) \rightarrow g g\right)=0.3-0.5$ [34 or $0.64 \pm 0.11$ [33]. Since the expectation for a glueball is that $B R(G \rightarrow g g) \geq 0.5$ [34], both $f_{0}(1710)$ and $f_{0}(1500)$ are consistent with a sizable glueball component, and with $f_{0}(1710)$ having a larger glueball component. Moreover, ref. [35 concludes that $f_{0}(1370)$ may have some glueball admixture, smaller than $f_{0}(1500)$ and $f_{0}(1710)$, but is dominantly quarkonium, partially in agreement with our results. Predictions here are complicated by the large width of $f_{0}(1370)$ [34].

\section{Summary}

In this paper we suggest a coherent view at the scalar glueball as having the following properties:

(i) A (physical) intermediate state in scalar $q \bar{q}$ annihilation-creation transitions (called "glueball dominance").

(ii) A state decaying to two mesons via an intermediate scalar meson.

(iii) A primitive state which mixes with the primitive $n \bar{n}(n=u, d)$ and $s \bar{s}$ quarkonia to form three physical scalar mesons.

Three main assumptions are employed in this work: glueball dominance, SU(3) symmetry and the assumption that only ground state quarkonia are relevant to scalar glueball mixing and decay.

As can be seen in Figure 1, glueball dominance together with the calculation of energy dependent couplings in the ${ }^{3} P_{0} /$ flux-tube model can account for the counterintuitive primitive glueball couplings to $\pi \pi, K \bar{K}$ and $\eta \eta$ found in lattice QCD. In Table 1 a total glueball width of greater than $250-390 \mathrm{MeV}$ with a dominant $a_{1} \pi$ decay of $70-180 \mathrm{MeV}$ is predicted. Decay to $(\pi \pi)_{S}(\pi \pi)_{S}$ may also be significant as was observed experimentally for $f_{0}(1500)$ and $f_{J}(1710)$.

The quadratic mass matrices in the $2 \times 2$ quarkonium and $3 \times 3$ glueball-quarkonium 
formulations are equivalent. The linear and quadratic $3 \times 3$ glueball-quarkonium mass matrices are equivalent under the requirements that (i) $z^{2} \ll m_{G}^{2}, m_{s \bar{s}}^{2}, m_{u \bar{u}}^{2}$ and (ii) $m_{s \bar{s}}-m_{n \bar{n}} \ll m_{n \bar{n}}, m_{s \bar{s}}$ or $m_{n \bar{n}}, m_{s \bar{s}} \ll m_{G}$. The conditions mentioned are always fulfilled in this work, and an illustrative example of the equivalence can be found in Appendix B.

The $f_{0}(1500)$ is not the heaviest state arizing from glueball-quarkonium mixing, implying that if the existence of both $f_{0}(980)$ and $f_{0}(1370) / f_{0}(400-1200)$ is confirmed, allowance should be made for an additional degree of freedom. The glueballquarkonium coupling extracted from our glueball decay analysis is consistent with estimates from lattice QCD.

The mass of the physical glueball is consistent with the experimental $f_{J}(1710)$. Experimental two-photon and $J / \psi$ radiative decay data are consistent with the valence content predicted for the physical states.

\section{Acknowledgments}

We wish to thank T. Barnes, V.M. Belyaev, D.V. Bugg, W. Dunwoodie, T. Goldman, N. Isgur, W. Lee and D. Weingarten for valuable discussions during the preparation of this work.

\section{Appendix A: Glueball decay couplings}

The glueball decay amplitudes (evaluated in Table 1) to various outgoing states are (for $\beta_{A}=\beta_{B}=\beta_{C} \equiv \beta$ and identical quark masses)

$$
\mathcal{M}=\frac{f_{S U(3) G}}{m_{S U(3)}^{2}-m_{\tilde{G}}^{2}} \sqrt{\frac{8 m_{G}^{2} \tilde{M}_{B} \tilde{M}_{C}}{\tilde{M}_{A}}} \sqrt{\frac{2}{\beta}} \pi^{\frac{3}{4}} \gamma_{0} \exp \left\{-\frac{p^{2}}{12 \beta^{2}}\right\} \varpi
$$

where

$$
\begin{array}{ll}
\varpi=\frac{2^{\frac{9}{2}}}{3^{2}}\left(1-\frac{2}{9}\left(\frac{p}{\beta}\right)^{2}\right)\left\{\begin{array}{cll}
1 & \text { for } \pi \pi, K \bar{K}, \eta \eta \\
\frac{1}{\sqrt{3}} & \text { for } \rho \rho, \omega \omega, K^{*} \bar{K}^{*} & \text { (S-wave) } \\
\varpi=-\frac{2^{7}}{3^{\frac{9}{2}}}\left(\frac{p}{\beta}\right)^{2} & \text { for } \rho \rho, \omega \omega, K^{*} \bar{K}^{*} & \text { (D-wave) }
\end{array}\right.
\end{array}
$$




$$
\begin{aligned}
& \varpi=\frac{2^{\frac{11}{2}}}{3^{3}} \frac{p}{\beta} \quad \text { for } a_{1} \pi \quad \text { (P-wave) } \\
& \varpi=\frac{2^{4}}{3^{\frac{7}{2}}}\left(1+\frac{19}{18}\left(\frac{p}{\beta}\right)^{2}-\frac{1}{27}\left(\frac{p}{\beta}\right)^{4}\right) \quad \text { for } \pi(1300) \pi \quad \text { (S-wave) } \\
& \varpi=\frac{2^{\frac{11}{2}} 5}{3^{4}}\left(1+\frac{19}{180}\left(\frac{p}{\beta}\right)^{2}-\frac{1}{270}\left(\frac{p}{\beta}\right)^{4}\right) \quad \text { for } f_{0}(600) f_{0}(600) \quad \text { (S-wave) }
\end{aligned}
$$

\section{Appendix B: Numerical example of the equivalence of linear and quadratic mass matrix formulations}

An illustrative example of how Proposition 2 works is the case analyzed by Weingarten 10 where for the input masses $m_{n \bar{n}}=1450 \mathrm{MeV}, m_{s \bar{s}}=1516 \mathrm{MeV}, m_{G}=1635 \mathrm{MeV}$ and $z=77 \mathrm{MeV}$, the masses of the three physical states are $m_{\tilde{G}}=1710 \mathrm{MeV}$, $m_{f_{0}^{\prime}}=1.5 \mathrm{GeV}$ and $m_{f_{0}}=1390 \mathrm{MeV}$. To translate this linear mass case into our mass squared one we use the relation $f=z\left(m_{G}+m_{q \bar{q}}\right)$ near Eq. (4.16) which, with $m_{q \bar{q}}=\left(m_{n \bar{n}}+m_{s \bar{s}}\right) / 2$, gives $f \simeq 0.24 \mathrm{GeV}^{2}$. When this $f$ and the same input masses squared are used in (4.2), we obtain the masses of the physical states, $m_{\tilde{G}}=1706$ $\mathrm{MeV}, m_{f_{0}^{\prime}}=1.5 \mathrm{GeV}, m_{f_{0}}=1383 \mathrm{MeV}$, which are in excellent agreement with Weingarten's case, and the valence content of the physical states,

$$
\begin{aligned}
|1706\rangle & =0.87|G\rangle+0.34|s \bar{s}\rangle+0.36\left|\frac{u \bar{u}+d \bar{d}}{\sqrt{2}}\right\rangle, \\
|1500\rangle & =-0.18|G\rangle+0.89|s \bar{s}\rangle-0.41\left|\frac{u \bar{u}+d \bar{d}}{\sqrt{2}}\right\rangle \\
|1383\rangle & =-0.46|G\rangle+0.29|s \bar{s}\rangle+0.84\left|\frac{u \bar{u}+d \bar{d}}{\sqrt{2}}\right\rangle
\end{aligned}
$$

which is in excellent agreement with the corresponding valence content of ref. [10]:

$$
\begin{aligned}
|1710\rangle & =0.87|G\rangle+0.34|s \bar{s}\rangle+0.36\left|\frac{u \bar{u}+d \bar{d}}{\sqrt{2}}\right\rangle \\
|1500\rangle & =-0.19|G\rangle+0.90|s \bar{s}\rangle-0.40\left|\frac{u \bar{u}+d \bar{d}}{\sqrt{2}}\right\rangle \\
|1390\rangle & =-0.46|G\rangle+0.28|s \bar{s}\rangle+0.84\left|\frac{u \bar{u}+d \bar{d}}{\sqrt{2}}\right\rangle .
\end{aligned}
$$




\section{References}

[1] G. Bali et al. (UKQCD Collab.), Phys. Lett. B309 (1993) 378

[2] H. Chen, J. Sexton, A. Vaccarino and D. Weingarten, Nucl. Phys. Proc. Suppl. 34 (1994) 357

[3] W. Lee and D. Weingarten, Nucl. Phys. Proc. Suppl. 63 (1998) 194

[4] W. Lee and D. Weingarten, hep-ph/9805029

[5] F.E. Close and M. Teper, OUTP-95-06P, OUTP-96-35P, RAL-96-040 (unpublished)

[6] Particle Data Group (R.M. Barnett et al.), Phys. Rev. D54 (1996)

[7] M.M. Brisudova, L. Burakovsky and T. Goldman, LA-UR-97-3794 hepph/9712514

[8] W.-S. Hou and C.-Y. Ko, hep-ph/9708314

[9] W. Lee and D. Weingarten, Nucl. Phys. Proc. Suppl. 53 (1997) 236

[10] D. Weingarten, Nucl. Phys. Proc. Suppl. 53 (1997) 232

[11] J. Sexton, A. Vaccarino and D. Weingarten, Phys. Rev. Lett. 75 (1995) 4563;

Nucl. Phys. Proc. Suppl. 47 (1996) 128; ibid. 42 (1995) 279

[12] W.-S. Hou, Phys. Rev. D55 (1997) 6952

[13] W.-S. Hou and A. Soni, Phys. Rev. D29 (1984) 101

[14] R. Kokoski and N. Isgur, Phys. Rev. D35 (1987) 907

[15] P. Geiger and E.S. Swanson, Phys. Rev. D50 (1994) 6855

[16] P.R. Page, Nucl. Phys. B446 (1995) 189

[17] N. Isgur and J.E. Paton, Phys. Rev. D31 (1985) 2910

[18] T. Barnes, F.E. Close, P.R. Page and E.S. Swanson, Phys. Rev. D55 (1997) 4157 
[19] C. Amsler and F.E. Close, Phys. Rev. D53 (1996) 295

[20] D. Weingarten, private communication

[21] J.Y. Cui, H.Y. Jin and J.M. Wu, hep-ph/9803276

[22] M. Boglione and M.R. Pennington, Phys. Rev. Lett. 79 (1997) 1998; M.R. Pennington, Proc. of HADRON '97' (Upton, N.Y., August 1997), p. 140, eds. S.-U. Chung, H.J. Willutzki

[23] N. Isgur and P. Geiger, Phys. Rev. D47 (1993) 5050; N. Isgur, JLAB-THY97-14, Proc. of 25 th INS Int. Symposium on Nuclear and Particle Physics with High-Intensity Proton Accelerators (Tokyo, Dec. 1996)

[24] L. Burakovsky and T. Goldman, Phys. Rev. D57 (1998) 2879

[25] J.Z. Bai et al. (BES Collab.), Phys. Rev. Lett. 77 (1996) 3959

[26] D.V. Bugg et al., Phys. Lett. B353 (1995) 378

[27] W. Dunwoodie, SLAC-PUB-7163; Proc. of HADRON '97 (Upton, N.Y., August 1997), p. 753, eds. S.-U. Chung, H.J. Willutzki

[28] R. Kamiński, L. Leśniak, K. Rybicki, Z. Phys. C74 (1997) 79

[29] U. Thoma (Crystal Barrel Collab.), Proc. of HADRON '97 (Upton, N.Y., August 1997), p. 322, eds. S.-U. Chung, H.J. Willutzki

[30] A. Abele et al. (Crystal Barrel Collab.), Phys. Lett. B380 (1996) 453

[31] T. Barnes, Proc. of $9^{\text {th }}$ International Workshop on Photon-Photon Collisions (San Diego, 1992), p. 263, eds. D.O. Caldwell and H.P. Paar, World Scientific; E. Klempt et al., Phys. Lett. B361 (1995) 160; C.R. Münz, Nucl. Phys. A609 (1996) 364

[32] G.D. Lafferty, Proc. of HADRON '97 (Upton, N.Y., August 1997), p. 80, eds. S.-U. Chung, H.J. Willutzki

[33] Z. Li, Proc. of "Intersections between Nuclear and Particle Physics" (Big Sky, 1997), p. 510, ed. T.W. Donnelly, American Institute of Physics 
[34] F.E. Close, G. Farrar, Z.-P. Li, Phys. Rev. D55 (1997) 5749.

[35] F.E. Close, Nucl. Phys. A623 (1997) 125c; Nucl. Phys. Proc. Suppl. 56A (1997) 248

[36] E.S. Swanson, Ann. Phys. 220 (1992) 73

[37] R. Gupta et al., Phys. Rev. D43 (1991) 2301; G.S. Bali et al. (SESAM Collab.), Nucl. Phys. Proc. Suppl. 63 (1998) 209; ibid. 53 (1997) 239; P. de Forcrand and K.-F. Liu, Phys. Rev. Lett. 69 (1992) 245; C.J. Morningstar and M. Peardon, Phys. Rev. D56 (1997) 4043

Fig. 1: The amplitude $\mathcal{M}$ (in $\mathrm{GeV}$ ) plotted against the square of the momentum in the outgoing state $p^{2}$ (in $\mathrm{GeV}^{2}$ ). The sold line is our basic prediction and the data points are the lattice predictions, both of which are discussed in the text. We allow the following parameter variations. (a) The dashed line differs from the basic prediction in that we take inverse radii motivated from studies of masses and wave functions of mesons and glueballs, not decays. The $\pi$ and $K$ inverse radii were estimated as $0.54 \mathrm{GeV}$ and $0.53 \mathrm{GeV}$ respectively [36], so we take $\beta_{B}=\beta_{C}=0.54 \mathrm{GeV}$. We also talk the glueball to have an r.m.s. radius $\sqrt{\left\langle r^{2}\right\rangle}$ of $\sim \frac{1}{2} \mathrm{fm}$ [1, 37]. Assuming that the scalar ( $\mathrm{P}$-wave) meson coupling to the glueball has the same size, one estimates for S.H.O. wave functions that $\beta_{A} \sqrt{\left\langle r^{2}\right\rangle}=\sqrt{\frac{5}{2}}$, yielding $\beta_{A}=0.6 \mathrm{GeV}$. (b) The solid black dots differ from the basic prediction, in that we adopt the relativistic phase space convention [18, 15]. Here we make the replacements $\tilde{M}_{A} \rightarrow m_{G}$ and $\tilde{M}_{B}, \tilde{M}_{C} \rightarrow \sqrt{m_{P S}^{2}+p^{2}}$ in Eq. (3.2), where $m_{P S}$ is the outgoing pseudoscalar mass. From left to right the points correspond to the $\eta \eta, K \bar{K}$ and $\pi \pi$ decay modes. The large points correspond to $\gamma_{0}=0.4$ [18], and the small points to $\gamma_{0}=0.53$ [15]. 


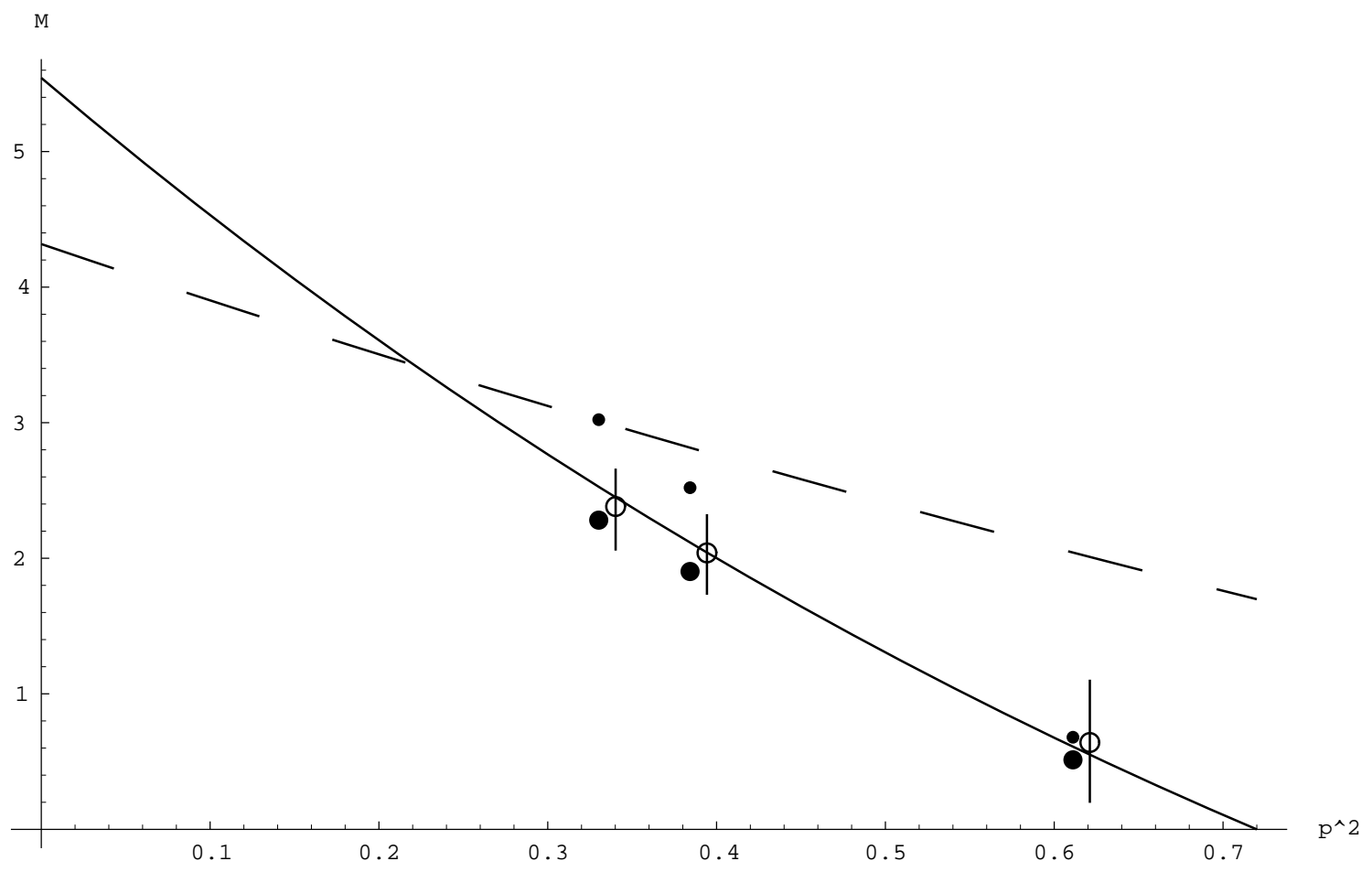




\begin{tabular}{|c|c||c|c|c|c|c||c|}
\hline Decay Mode & Wave & $1.4 \mathrm{GeV}$ & $1.5 \mathrm{GeV}$ & $1.6 \mathrm{GeV}$ & $1.7 \mathrm{GeV}$ & $1.8 \mathrm{GeV}$ & Width $(\mathrm{MeV})$ \\
\hline$\pi \pi$ & $\mathrm{S}$ & 1.31 & 0.96 & 0.55 & 0.093 & -0.41 & $6(6)$ \\
$K \bar{K}$ & $\mathrm{~S}$ & 2.8 & 2.7 & 2.0 & 1.6 & 1.0 & $81(82)$ \\
$\eta \eta$ & $\mathrm{S}$ & 3.2 & 2.9 & 2.4 & 2.0 & 1.4 & $27(27)$ \\
$a_{1} \pi$ & $\mathrm{P}$ & $10.7 p$ & $11.2 p$ & $11.7 p$ & $12.0 p$ & $12.3 p$ & $177(67)$ \\
$\pi(1300) \pi$ & $\mathrm{S}$ & & 1.1 & 1.4 & 1.8 & 2.3 & $23(7)$ \\
$\rho \rho$ & $\mathrm{S}$ & & & 3.3 & 3.0 & 2.6 & $61(46)$ \\
$\omega \omega$ & $\mathrm{S}$ & & & 3.5 & 3.1 & 2.7 & $16(12)$ \\
$K^{*} \bar{K}^{*}$ & $\mathrm{~S}$ & & & & & 4.1 & \\
$(\pi \pi)_{S}(\pi \pi)_{S}$ & $\mathrm{~S}$ & $<9.6$ & $<10.2$ & $<10.9$ & $<11.5$ & $<12.1$ & $<490(<160)$ \\
\hline
\end{tabular}

Table 1: Amplitudes for the decay of a scalar glueball to two mesons in GeV. For $\mathrm{P}$-wave decays the linear momentum dependence is explicitly separated (with $p$ in $\mathrm{GeV}$ ). The amplitudes for a $1.6 \mathrm{GeV}$ glueball should be regarded as our predictions for the primitive glueball. The other amplitudes are to be used for calculation of the decays of the physical states. The widths (including all partial waves) are listed in the final column for a $1.6 \mathrm{GeV}$ glueball, assuming that all resonances are narrow. All calculations are for mock meson phase space, except the widths in brackets in the last column, which are for relativistic phase space with $\gamma_{0}$ chosen to agree with lattice QCD predictions for glueball decay to $\pi \pi, K \bar{K}$ and $\eta \eta$. When both $\mathrm{S}-$ and D-wave amplitudes are possible the amplitude is the $\mathrm{S}$-wave amplitude. The ratio (D-wave amplitude) $/\left(p^{2} \mathrm{~S}\right.$-wave amplitude) is -4.2 and -4.1 for decays of a $1.6 \mathrm{GeV}$ glueball to $\rho \rho$ and $\omega \omega$, respectively, and -4.0 for decay of a $1.8 \mathrm{GeV}$ glueball to $K^{*} \bar{K}^{*}$. $(\pi \pi)_{S}$ stands for a (hypothetical) narrow $(u \bar{u}+d \bar{d}) / \sqrt{2}$ resonance $f_{0}(600)$ which decays dominantly to $\pi \pi$ [6]. It may be related to the low mass tail of the $f_{0}(1370) / f_{0}(400-1200)$. Due to the large width and uncertain mass of the physical $(u \bar{u}+d \bar{d}) / \sqrt{2}$ scalar resonance, the predictions should be viewed as being anywhere between zero and the upper limit quoted. 